\title{
Role of Navigation in Oral and Maxillofacial Surgery: A Surgeon's Perspectives
}

This article was published in the following Dove Press journal:

Clinical, Cosmetic and Investigational Dentistry

\section{Manish Anand (D) \\ Shreya Panwar}

Department of Oral and Maxillofacial Surgery, Meenakshi Ammal Dental College, Chennai, Tamil Nadu, India
Correspondence: Manish Anand Department of Oral and Maxillofacial Surgery, Meenakshi Ammal Dental College, Alapakkam, Chennai, Tamil Nadu, 600056, India

Tel +9l 805624658।

Email manishanand028@gmail.com
Abstract: Surgeries related to the maxillofacial area deal with an intricate network of anatomical structures. With the complexity of the vital structures, it necessitates a surgical team to respect each anatomical boundary. In the past, there was an exceptionally high number of cases with surgical errors. These errors were not because of flaws in the surgeon's skills or techniques but owing to lack of resources. Visualisation is one of the key factors that determines the precision of any surgical outcome. Advances in surgical planning have led to the introduction of a "Navigation" system that helps surgeons to see more, know more and ultimately do more for their patients. The usefulness of the navigation system in oral surgeries has been indicated by its surgical applications in craniomaxillofacial trauma, orthognathic surgeries, head and neck pathological resections, complex skull base surgeries and surgery involving temporomandibular joint. A vast majority of research literature has suggested remarkable improvement in surgical outcomes under the guidance of $3 \mathrm{~d}$ planning and navigation. However, with such an inordinate advancement, financial expenses and a gradual learning curve are always a constraining factor in surgical navigation. This article overviews indication of navigation in craniofacial surgeries with a focus on applied aspect, planning and solution to the future problem.

Keywords: navigation, computer-assisted surgeries, craniomaxillofacial, orthognathic, trauma

\section{Introduction}

Over the last few years, technological advancement has significantly impacted surgical outcomes. From knife to robotic surgeries, and from $2 \mathrm{~d}$ imaging to $3 \mathrm{~d}$ imaging, technology has become an integral part of any surgery. Navigationassisted surgery is one such example of a technological boon applied to medicine. In simple term, navigation means a device that can accurately locate critical anatomical structures, the safest way to reach that target, and the orientation around which safe and reliable surgery can be carried out. It helps surgeons unlock an "out of reach" area that is impossible to access with traditional imagining techniques. Over time, this masterpiece has evolved into a robust technology that enables surgeons to perform more challenging surgeries, which were once considered to be unfeasible and impractical.

Neurosurgery was the first domain that blended navigation in their procedures. The brain is the most delicate organ of the human body, and since time immemorial, surgeons have tried to develop new techniques in their quest for performing minimally invasive surgeries around this area. This led to the discovery of navigation, and stereotaxy was the first neurosurgical procedure carried out under the 
guidance of navigation. ${ }^{1}$ With further refinement, this technology was further incorporated into other areas and specialities of surgery. Of late, with better understanding, Oral and Maxillofacial surgeons have started planning critical surgeries using this technology. It first started with the correction of secondary deformity, and soon it collaborated with other branches of maxillofacial surgeries.

Shreds of evidence from the literature have substantiated the superiority of using the Navigation system over other imaging modalities. Dubois et al, in the year of 2015, studied the final implant position in 10 cadaveric models secondary to orbital trauma and concluded that navigation assisted surgeries had a positive impact on the desired results. Their research showed that using navigation technology in maxillofacial pathology surgery increases predictability by facilitating accurate safety margins and protecting vital anatomical boundaries. ${ }^{2,3} \mathrm{Wu}$ et al in 2019, with their systemic review, vouched for real-time navigation surgery during zygomatic implant placement secondary to mid-face trauma. ${ }^{4}$ Although research literature has always supported the navigation system's positive outcome, clinical study on a large patient group is still lacking, and therefore, it will not be wise to grant more dominion to navigation over other time-proven and tested systems.

Much has been spoken about $3 \mathrm{~d}$ planning and navigation surgeries; understanding "how navigation works?" requires understanding at the grassroots level. This article has focused more on planning and designing a workflow to understand this complex mechanism on an easy ground level with the evidence from the existing literature.

\section{Concept of Navigation}

The surgical navigation system works exclusively on the Global Positioning System (GPS) principle that synchronises location and time data from point $\mathrm{A}$ to point B. A Surgical Navigation System is made up of three primary components - a localiser, which is analogous to a satellite in space; Computerised tomography (CT) scan data, which is analogous to a ground control or road map; and a surgical probe in the Navigation system, which is analogous to the user equipment. ${ }^{5}$

In GPS, a satellite works as a fixed frame of reference, which sends signals that are read and interpreted by the GPS equipment fixed in a user device. Once this signal is picked up by a sensor probe (attached to a smartphone or smart-watch), it is converted into microwave signals to determine the location from that fixed point of reference. In navigation-guided surgeries, this satellite or localiser is fixed on the patient's forehead and emits signals which are then picked up by a surgical probe and then converted into a digital image (Figure 1). This digital image is then picked up by a monitor that orients the patient's present spatial position on a pre-registered CT or Magnetic Resonance Imaging (MRI) file.,

\section{How Navigation Works?}

Navigation assisted surgeries to undergo the following six main steps -

1. Data acquisition

2. Mirroring

3. Preoperative surgical planning

4. Registration Marker

5. Execution of planned surgery

6. Final check

The obtained CT data is first converted into the Digital Imaging and Communications in Medicine.

(DICOM) images, which are then imported into the planning software. Segmentation of the obtained images is done based on the Hounsfield unit to differentiate between soft and hard tissues.

Once the data is uploaded onto the system, the next step is to mirror the defective side to its contralateral normal side. Hence, this step allows surgeons to measure critical defect volume, and the reconstructive implant is then 3-d printed according to the known defect volume. In resection surgeries, virtual surgery is initially carried out before mirroring, where software guided control allows surgeons to remove pathological mass after calculating anatomically safe margins 3-dimensionally. After removing the pathological mass, the formed defect is then mirrored to the contralateral side, and the final template is prepared, based on which reconstructive implants are 3-d printed.

Pre-surgical planning software shares the control of data presentation from the radiologist to the surgeon. He/ she can modulate and analyse the virtual model on a 3-D axis with freedom. This offers an accurate prediction of the depth, width, and projection. The surgeon can assess bony defects or deformities that would otherwise be very difficult to see in a 2-D image. ${ }^{7}$

As mentioned above, the first three steps (1-3) are carried out before surgery, while the next three steps (4-6) are performed in the operation theatre. Registration of point is the most crucial step in navigation surgery, 


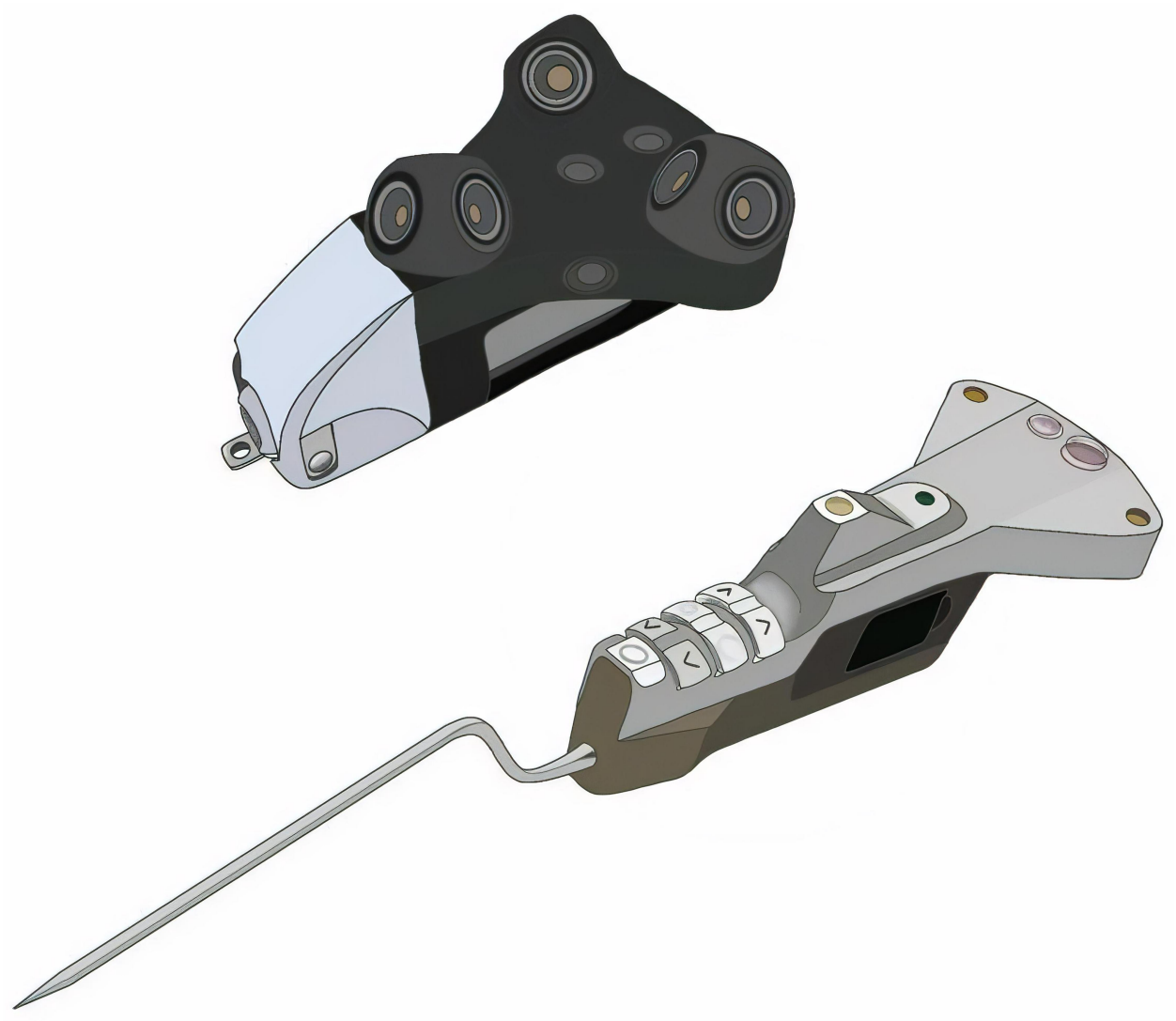

Figure I A localizer (top) and a surgical probe or tracker (bottom) - Technical part of a navigation system. A localizer is fixed on patient forehead and a surgical probe or tracker guides surgeon for point registration.

carried out by a sensor probe or tracker attached to a navigation system $[5,6]$. Generally speaking, these points are the bony landmarks, which serve as guiding points to predict critical anatomical landmarks and also to orient the surgical plane based on these references (Figures 2 and 3). Although these reference points can vary according to the

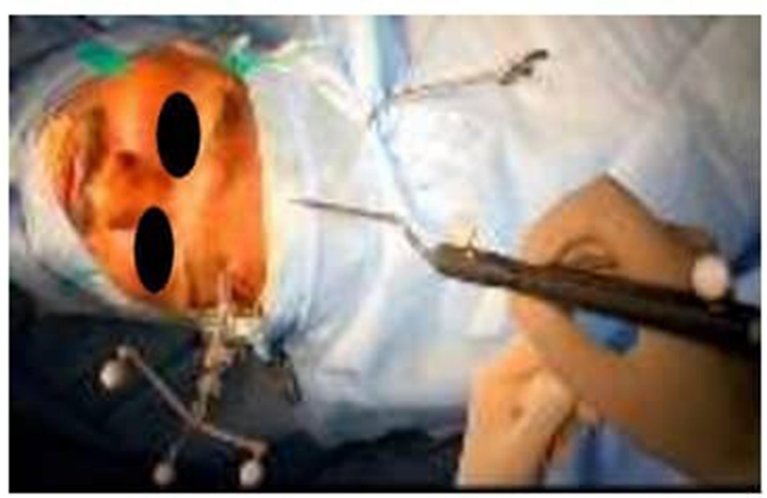

Figure 2 Intra-operative placement of localizer and a surgical probe. A pointed end of surgical probe serves a purpose of marking anatomical landmark. surgical requirements, the commonly used points of reference are: frontonasal junction, the medial and lateral rim of the orbit, the superior and inferior rim of the orbit, zygomatic prominence, the tip of the nose, angle of the mandible and chin point. These points are marked just before an incision and are then registered into the navigation software, reflecting the registered points on to the monitor. $^{8}$

Surgical exposure is done as per planning, and the sensor probe matches the area of interest. A confirmation point is then obtained on the screen by matching preoperative landmarks to the intraoperative points. The surgeon can now see two lines coinciding in all three planes (sagittal, coronal and axial). ${ }^{8,9}$ This verifies the correct plane of dissection. During reconstructive surgery or where implant placement is needed, the final check is carried out after implant placement and before fixation. For example, in orbit reconstruction, a mesh must fall on the poster-o-lateral ledge of the junction of the medial and the inferior orbital wall. ${ }^{10}$ Once the implant placement is done, the junction where the implant should lie is checked 


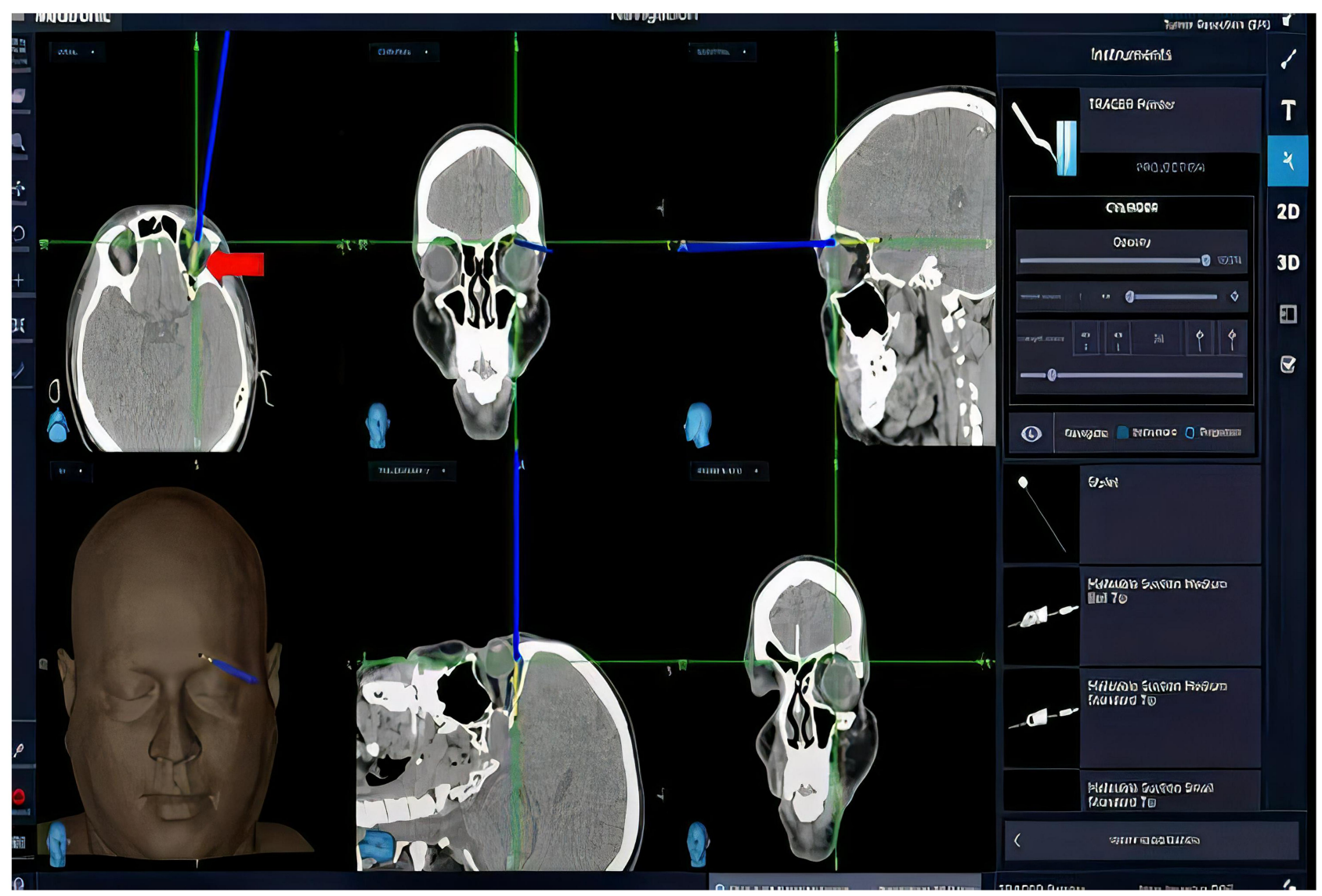

Figure 3 Intraoperative verification of points in axial, coronal and sagittal section. A red arrow indicate the supraorbital bony landmark verified by the confluence of line during point registration with a navigation probe.

on all three planes, which are generally shown by two lines coinciding with each other. Once this is verified, fixation and closure are then carried out subsequently.

\section{Application in Oral and Maxillofacial Surgeries}

Although neurosurgeons were the first to introduce navigation technology in their practice, it was not until 2002, when a surgical team led by Alexander Schramm performed navigation guided surgery of the Temporomandibular joint to remove the ankylotic mass. ${ }^{11}$ In the procedure, they reported success with great precision in identifying anatomical structure, which marked the beginning of navigation in complex maxillofacial procedures. With time and better understanding, surgeons were able to gain more insight into navigation and started experimenting with other procedures. This system soon emerged as one of the most reliable inventions gifted to surgery by technology, and it continues to excel surgical interventions into safer and less invasive procedures. ${ }^{1}$ At present, Navigation guided surgery has become one of the most critical components of any complex surgeries of the head and neck. With current research and further software modification, this technological boon will soon become the backbone of craniomaxillofacial and plastic surgery.

\section{Clinical Application in Maxillofacial Trauma}

One of the areas where navigation is widely used is trauma involving the upper and middle third of the face. Zygomatic bone serves as a corner keystone for facial buttress. Any trauma leading to this region results in facial disproportion and disfigurement. ${ }^{12}$ Many a time, the zygomatic-maxillary complex has such a complicated anatomy that even after adequate treatment, it may result in secondary deformities that are aesthetically distressing for patients. A study by Ellis et al found that disparity of $2 \mathrm{~mm}$ bilateral facial differences is considered visually symmetrical. Interestingly, Xi Gong et al in one of his studies concluded that bilateral facial difference was in the range of $1.24-1.36 \mathrm{~mm}$ in a navigation-assisted surgery group while it was 
significantly higher in a manual reduction group $(2.24 \mathrm{~mm}-$ $3.60 \mathrm{~mm}$ ), which were visually perceptible as increase in the transverse width of the face. ${ }^{14}$ Navigation allows us to accurately replicate the model of the uninjured side, which is then mirrored onto the injured side. The differences in defect volume are then calculated and a virtual implant is printed, based on these differences. Theoretically, since the injured side is mirrored onto the uninjured side, it helps in achieving near similar symmetry. Once this symmetrical bony framework is achieved, at a later stage soft tissue correction can be done by an autologous fat graft or silicone mesh.

Another very challenging surgery that is quite difficult to correct is trauma involving Nasoorbital-ethmoid complex. Trauma to this region may lead to aesthetically debilitating conditions such as late Tele canthus, enophthalmos, proptosis, dystopia, etc. ${ }^{15}$ Injury to the orbit significantly causes fracture line to propagate through the inferior orbital wall (since it is the weakest zone of the orbit), leading to inferior displacement of the orbital floor, which results in enophthalmos. Enophthalmos greater than $2 \mathrm{~mm}$ is a prime indicator for the orbit reconstruction which causes significant increase in the orbital volume. A difference of 3-4 $\mathrm{mm}$ between the position of the globe is clinically visible as marked posterior retro positioning of the globe. To correct this, surgeons usually place a titanium mesh, thus recreating the orbital floor to support the globe. The implant must exactly lie on the confluence area, ie, the anatomical junction of the convergence of the medial and posterior walls of the orbit which is approximately $22 \mathrm{~mm}$ from the inferior orbital rim. ${ }^{16}$ Earlier surgeons used to reconstruct this defect, based on the clinical manipulation and CT findings, which resulted in inaccurate placement of implants, and the desired outcomes were never achieved. The confluence zone is inaccessible, and almost impossible to visualise with the naked eye; but with the advent of navigation systems, an operating surgeon can first superimpose these defects and print a patientspecific implant. Consequently, he/she can visualise the confluence zone on the monitor, and determine the final prosthetic (Figure 4).

Upon inspecting sources in literature, we find a study conducted by Yu et al in 2013, where they enrolled thirty four patients with zygomatic-orbital complex fracture, which were planned to be operated under navigation guidance. ${ }^{17}$ They concluded that navigation-assisted surgeries are quite helpful in precise anatomical reduction, asymmetry correction, and safe manipulation along the delicate anatomical structure. Another study conducted by Jia-Ruei Yang et al in 2019, where they retrospectively analysed 17 patients with orbital complex fracture that were treated under the guidance of navigation between 2015 and 2017, concluded that there was an average reduction of enophthalmos from $2.99 \mathrm{~mm}$ to $0.68 \mathrm{~mm}$, with no post-operative complications; and all the patients were satisfied with their final appearance and function. ${ }^{18}$ A major study conducted by He et al from the year 2008 to 2010, where they involved 64 patients with delayed presentation of orbito-zygomatic fracture, concluded that Navigation-assisted surgery with a 3D model and titanium mesh are the best ways to manage delayed orbitozygomatic fractures with gross enophthalmos. ${ }^{19}$ A similar study by $\mathrm{Yu}$ et al further confirmed the accuracy of

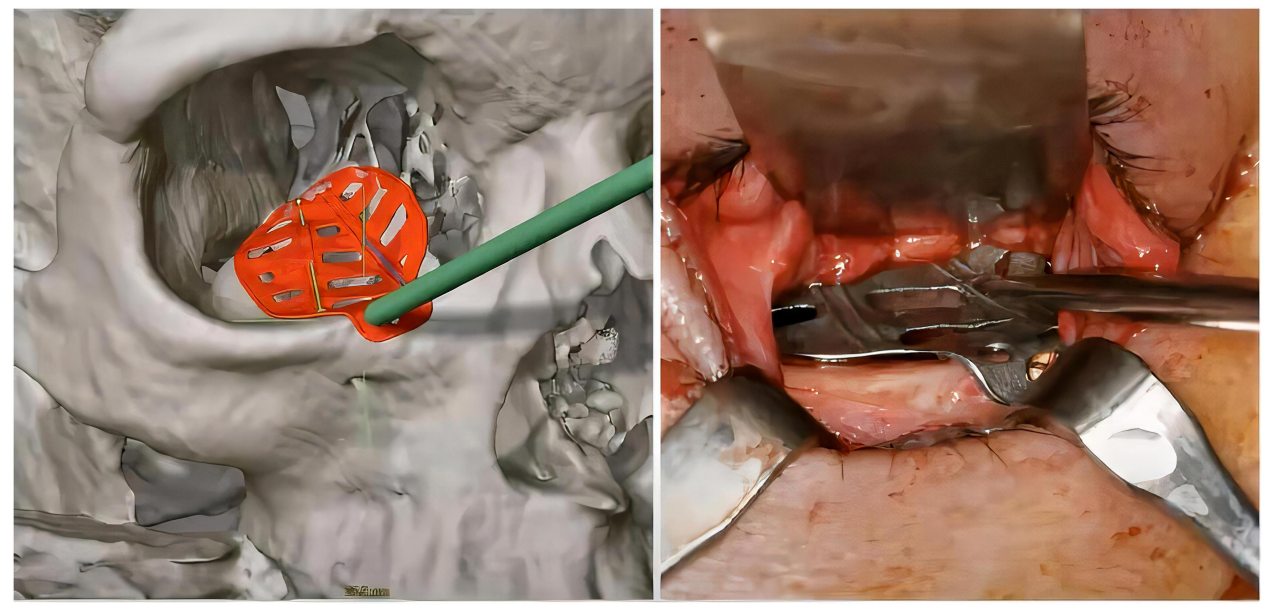

Figure 4 Pre-operative verification of orbital mesh (left) using navigation. Reproducing the plan during surgery under the guidance of navigation (right). 
navigation by concluding error of less than $1 \mathrm{~mm}$ between the registration of intraoperative anatomy with preoperative CT scans. He further noted a maximum deviation of less than $2 \mathrm{~mm}$ when comparing post-operative $\mathrm{CT}$ scans with pre-operative treatment planning. ${ }^{20}$

\section{Clinical Application in Orthognathic Surgeries}

Using navigation in orthognathic surgery gives us the benefit of clear visualisation of the critical anatomical structures and allows the surgeon to gain accurate access under guidance. Once access is obtained, it becomes easy for the surgeon to orient his/her surgical instruments accordingly. The final outcome of any orthognathic surgery depends mainly on how a surgeon analyses the region's relevant anatomy. One of the complicating factors of these procedures is that the relevant structures are located deep, and it requires extreme dexterity to operate around these areas. Therefore, it is critical to have an insight into the anatomical features and positional relationships of each patient. ${ }^{5}$ Furthermore, as the critical structures are accurately monitored during surgery, the procedure can be performed within safe margins.

The most common procedure performed on the lower jaw to correct the anteroposterior discrepancy is sagittal split osteotomy, followed by ramus osteotomy. In both these procedures, accurate identification of the inferior alveolar nerve's course is mandatory ${ }^{21}$ because neurosensory disturbances secondary to these osteotomies are very common. Under the navigation system's guidance, the accurate position of the tip of any surgical instrument can be monitored while the instrument is being used. ${ }^{5}$ With navigation, surgeons can precisely locate the position of an oscillating saw in relation to the lingual nerve and sigmoid notch during vertical ramus osteotomy - thus reducing the chances of damage to the neurovascular bundles. $^{5,22}$

In Maxilla, the most common procedure performed is Le fort 1 osteotomy, which requires the correct position of the pterygoid osteotome to prevent any unwanted complication in relation to the down fracture of the Maxilla. The Le Fort 1 osteotomy's most dreadful complication is - bleeding secondary to an injury to the internal maxillary artery and pterygoid venous plexus. ${ }^{20}$ Past reports have also demonstrated cases of blindness following Le fort 1 down fracture, as an excessive force created during down fracture travels through the pterygopalatine fissure to the optic nerve. With the navigation, surgeons can precisely orient the pterygoid osteotome at the junction of the pterygoid plate to create a neat disjunction of Maxilla from the pterygoid plates with minimum trauma to the surrounding structures. ${ }^{13}$ This precision, delivered by navigation technology, reduces the chances of unintentional injuries during these procedures.

Another salient feature of navigation-guided surgeries is the use of virtual 3D planning software, which allows surgeons to reproduce preoperative plans intraoperatively. Manipulation of the osteomised segments with final splints and the fixation of the osteomised segments can be planned pre-operatively. This allows surgeons to place maxillary and mandibular segments in a more ideal and favourable position. ${ }^{5,9}$ An error of $2 \mathrm{~mm}$ while placing an intermediate and a final splint may significantly cause midline shift and deranged occlusion.

In Distraction Osteogenesis, an incremental bone is allowed to grow. Using the Navigation system here enables the surgeon to point out the osteotomy sites accurately, guide the placement of screw holes to the planned site for the distractor, and align the vector of the distractor along with the planned site. ${ }^{23}$ Moreover, navigation surgery can also be used to predict the segment's vectorial movement before planning distraction osteogenesis. Access to this information and evaluating these positions using navigation allows more meticulous intraoperative verification of the expected bony changes. ${ }^{5}$

On reviewing sources from past literature, we find, Badiali et al conducted a retrospective study on 15 patients treated for asymmetric dentofacial deformities between 2010 and 2012 and concluded that simulation-guided navigation makes error-free post-operative outcomes with a mean deviation of less than $1 \mathrm{~mm}$ in preoperative and post-operative point-based and $3 \mathrm{~d}$ surface analysis in maxillary repositioning with the navigation system. ${ }^{24}$ Another study by Shiba et al on 46 patients, who underwent orthognathic surgery for the correction of dentofacial deformities, found that navigation-guided surgeries ease the accurate location of the pterygopalatine junction during maxillary osteotomies. $^{25}$ Based on author experiences and the study done in the past, we can conclude that navigation guided surgery has a clear cut role in planning orthognathic surgeries and also helps to verify surgical movements post-operatively

\section{Clinical Application in Skull Base Surgery}

Skull-based surgeries are among the most challenging surgeries because of their minimal accessibility and the 
complicated network of anatomical structures. Many a time, surgeons have to combine these procedures with an endoscope, but it needs extreme precision and agility. Because of the lack of a clear visual field, surgeons often leave the remnants of tumour cells, which might lead to recurrence later. The location, invasion, and margins of the tumour are the deciding factors in selecting surgical techniques. In the past, malignant tumours beyond the accessible areas, such as those located in the middle of the skull base or the infratemporal fossa, were considered inoperable due to hard-to-reach negative margin tumour cells and to achieve haemostasis in the event of massive haemorrhage. $^{26}$

However, with the introduction of Navigation, safer and quicker access to the skull base is now possible. Also, the extent and orientation of bone drilling can be planned pre-operatively so that the surgeon can work under a "safety net" intra-operatively. The demand for accuracy in skull base surgery is pervasive, and it requires an error of less than $0.5 \mathrm{~mm}$ for a safe procedure. ${ }^{27}$ Multiple studies have demonstrated the accuracy of Navigation in submillimetre in clinical and in the laboratory. ${ }^{28,29}$ In tumour surgery, the navigation system's use reduces the need for resection of vital structures and provides a clear image of the anatomical relationship with lesions at the skull base. Tumours involving the posterior and the lateral skull base may need an additional endoscopic visualization, as these areas are almost impossible to reach. ${ }^{11}$ Navigation is combined with an endoscope to manage sellar/parasellar lesions, benign pituitary lesions, and tumours encroaching the skull base like inverted papilloma, expansile myectomas, etc. Navigation in the skull base area is an upcoming technique, and the few clinics that are equipped with this technology have to rely on endoscopic visualization. Reviewing past literary sources, we can conclude that Navigation is beneficial, especially in operating benign tumours of the skull base, as it has a clear advantage over the endoscope in demarcating bony and soft tissue margins. ${ }^{30-32}$

\section{Clinical Application in Biopsies and Minimally Invasive Surgeries}

In biopsies of the head and neck region, Navigation first set its foot in and later established itself in the frameless stereotaxy domain. Since lesions in and around the skull base and pharyngeal space are difficult to access, it might be hard to obtain adequate tissue for histopathological sampling. ${ }^{5,30}$ Often, these lesions are so deep-seated that it mandates the use of general anaesthesia. Further, there is also a risk of injury to vital structures, owing to the lesions' hard-to-access location. ${ }^{33}$ Ultrasound-guided core needle biopsy is an excellent adjunct technique in diagnosing cervicofacial tumour masses because of its simplicity, safety, and minimally invasive nature. However, it is not viable for the skull base region and deep-seated lesions due to osseous intervention and reduced visibility.

With the advent of Navigation, the surgical team can first determine the lesion's extent in a 3-dimensional view, based on CT data incorporated into the navigation software. The trajectory of the biopsy needle is then planned. It offers a two-way advantage to the surgeons. First, the needle trajectory can be oriented towards the path of the least vital anatomical barrier, and second, safe margins can be delineated so that surgeons get a clear idea of the extent of the tumour when carrying out the final resection surgery. ${ }^{34}$ Furthermore, there is no potential risk of radiation hazard, as with CT- or MRI-guided biopsy. Also, Navigation can be quite useful in previously operated areas, which lack a clear surgical plane, because they had been compromised during the first intervention. In such cases, Navigation provides surgeons with a clear demarcation between the vascular and avascular planes. For further knowledge, readers are advised to refer to the study by Yang et al, who reported a 90\% diagnostic accuracy, across five years, in skull base and parapharyngeal pathological lesions. ${ }^{35}$

\section{Clinical Application in Tumour Reconstructive Surgery}

The principle of reconstruction directs us to - reconstruct with the same material that has been lost. Reconstruction of a lost structure secondary to tumour resection determines any oncological surgery outcome - both aesthetically and functionally. Early with limited availability of technology, surgeons' prime focus was to give back to the patient more of the functional component while compromising the aesthetic needs. With the introduction of sophisticated tools, this trend is changing. Today, the aesthetic, as well as the functional needs of the patients, are of prime concern for surgeons. With navigation, surgical margins are identified, and defect volume in all three planes is calculated pre-operatively. Based on these calculations, surgeons can design grafts more accurately. ${ }^{36,37}$ Although autogenous graft works well in reconstruction, 
the viability of graft and resorption of grafts following a surgery - is always under question. ${ }^{38}$ To overcome this, surgeons now prefer an alloplastic mode of reconstruction - mainly using titanium mesh or plate. As previously mentioned, navigation can accurately determine the defect volume. Using this, a surgeon can print a patient-specific and defect-specific titanium implant that will accurately fit into the void created after resection. To give perfect symmetry, surgeons can opt for soft tissue graft, mainly fat graft, by calculating differences in defect from the contralateral normal side.

\section{Clinical Application for the Removal of Foreign Body}

Removal of foreign bodies from the maxillofacial region is quite a daunting task because of their proximity to vital structures and inadequate visual access. In a high impact trauma, like a gunshot or in case of blast injuries, there is a high chance of the foreign body (bullet shell or kernel) getting embedded into the deeper structures. ${ }^{5}$ The aberrant anatomy of the region makes it more difficult to remove it safely. Although pre-operative CT scan with an image enhancer can correctly locate these foreign bodies, exploring intra-operatively without determining the correct plane may be challenging for surgeons. Also, the risk of damaging vital structures is relatively high while scouting in an incorrect plane. ${ }^{5,8}$ This, in turn, may lead to catastrophic postoperative morbidity. Navigation offers two-way benefits to surgeons in such scenarios - not only does it assist surgeons to locate and retrieve foreign bodies, but it also opens up scope for an immediate reconstruction - thereby reducing the treatment time and cost at the same time.

The technique for removing a foreign body with the aid of navigation follows the same principle as other navigation-aided surgeries. Once the foreign body is located preoperatively with CT imagery, navigation is used to assess that particular point with a sensor probe. The exact location is not confirmed until the lines from the two views (CT and navigation) coincide. ${ }^{39,40}$ Once the point is confirmed, surgeons then orient their instruments along that created plane, and blunt dissection is carried out until the foreign body is encountered. Surgeons have started using the navigation to retrieve foreign bodies from the maxillofacial region, which can be demonstrated from sources in the existing literature. A study by Sießegger et $\mathrm{al}^{41}$ on patients with a complication of foreign bodies embedded in the head and neck region concluded that foreign bodies were removed successfully with minimum intervention with the help of navigation. Also, it reduced the surgical time by more than $40 \%$ compared to similar interventions using the conventional technique. ${ }^{41}$ Reviewing the past case series and case reports in the literature shows that intra-operative navigation has clear supremacy over other locating and mapping modalities in terms of surgical access. Navigation also reduces intra-operative time and boosts postoperative recovery. ${ }^{39-45}$

\section{Clinical Application in Dentoalveolar Surgery}

Many a time, surgeries in and around the oral cavity become laborious for surgeons because of the limited access and the complex neurovascular anatomy. The most routinely performed procedures, where Navigation can be used, remove an unerupted tooth and implant surgeries. Determining the unerupted tooth's exact position that warrants extraction is the most crucial step in planning the surgery. With the incorporation of Navigation, a surgeon can first have a clear visualisation of the anatomical boundaries. This enables ease of removal of the tooth along the path of least resistance. For instance, removing a maxillary impacted third molar always carries a risk of displacement of roots in the maxillary sinus, fracture of the maxillary tuberosity and displacement of the tooth as a whole in potential spaces. ${ }^{46}$ Similarly, a mandibular third molar poses a risk of injury to the lingual and inferior dental nerves, displacement of a tooth into lingual spaces, and iatrogenic fracture of the mandible. ${ }^{47}$ Navigation system allows a surgeon to track down important boundaries within six degrees and also to calculate safe distance from these boundaries continuously. ${ }^{48,49}$ Once these points are tracked down, and a surgeon can then orient a surgical handpiece into the desired path to accomplish the rest of the surgical steps. These unerupted teeth are often associated with pathological lesions like a dentigerous cyst or an odontogenic keratocyst, which warrants extreme caution to safeguard vital anatomical the surgical plane lacks an apparent plane of usual structures. Navigation-assisted surgery in these scenarios is quite helpful, as it allows the operator to delineate and differentiate between the soft pathological mass and the hard tissue and preserve the neurovascular bundle.

Another commonly performed dentoalveolar surgery is implant surgery for a missing tooth or teeth 
replacement. The most critical steps in implant surgeries that determine the surgeries' fate are - the placement of the initial osteotomy drill, angulation of implant corresponding to the angulation of the surrounding natural tooth, optimum implant-bone-soft tissue interface, and excellent clearance from the relevant anatomical structures. ${ }^{48,50,51}$ Navigation-guided surgery enables surgeons to implant in more ideal conditions than traditional freehand implant surgeries. A study by Aydemir and Arisan et al noted that maximum acceptable deviation of less than $9 \mathrm{~mm}$ linear and 20.42-degree axial angulation was yielded by freehand execution. On the contrary, Navigation guided implant surgeries provided a maximum deviation of just $0.7 \mathrm{~mm}$ linear and 5-degree axial angulation. ${ }^{52}$ This technology allows surgeons to precisely locate implant placement in bone in all the 3-dimensions, without even opening a flap. With a tracker attached, the surgeon registers the relevant anatomical region that guides the operator to drill the initial osteotomy cut. A colour-coded depth indicator (attached to the system) helps the operator navigate and reach the targeted site. A colour change from green to yellow indicates that the drill bit is precisely $0.5 \mathrm{~mm}$ from the ideal site. ${ }^{48}$ On the navigation screen, the operator can virtually plan the implant's angulation to the corresponding dentition, further guiding the surgeon to drill along the desired plane angle. A major advantage of this system is that the surgeon can continuously monitor the procedures during the surgery. All the anatomical landmarks with ideal orientation are visualized on the screen so that the implant is placed in a more biologically and mechanically compatible environment, which ensures minimum error and better post-operative results.

The accuracy of implant surgeries guided by Navigation can be further verified by the study of Elian et al, who reported a mean linear accuracy ranging between $1.1 \mathrm{~mm}$ and $1.45 \mathrm{~mm}$ at the implant neck region with a mean angular deviation of 2 degrees. ${ }^{53}$ Another clinical study by Siessegge et al, where they placed 18 dental implants on a compromised site, concluded that an image-guided navigation surgery proved to be superior to conventional implant surgery in all practical aspects. ${ }^{54}$ Although much have been spoken about the accuracy of Navigation over conventional surgery, a recent study by $\mathrm{Wu}$ et al concluded that there are no significant statistical differences between Navigation assisted surgeries and conventional ones in terms of accuracy and postoperative outcomes. $^{55}$
Reviewing the best possible evidence from literature, it can be concluded that although navigation guidance is a useful adjunct in complex cases, it is not obligatory for experienced surgeons. In other words, Navigation helps surgeons to perfect the surgery, but experienced surgeons have often managed to deliver similar perfect results even without using Navigation.

\section{Limitations of Navigation}

Navigation technology has emerged as one of the most promising tools in the field of surgery, which has significantly changed the dynamics of intra-operative surgical accessibility and visibility. However, current navigation technology has its limitations, which prevent its application in specific scenarios. A crucial drawback of navigation technology is its inability to monitor dynamic movements continually and provide surgeons with a single static frame of reference. For example, surgeries involving the upper and middle thirds of the face - are relatively easy to perform - because the structures are in a fixed frame of reference concerning the cranial base. If the lower third is also involved with the upper or midface, it is not easy to synchronize with pre-operative CT scan since the mandible is free to move in all three planes. ${ }^{5,17,48,49,56}$ In these cases obtaining a single frame of reference becomes quite challenging for surgeons, and they have to depend on manual dexterity instead. Few authors have suggested the use of special sensors that will be fixed onto the mandible, which will guide surgeons to track the changes in jaw position intra-operatively continuously. ${ }^{48}$

Another limitation of the navigation technology is seen in the cases that require a bilateral operation. This is because navigation works on the principle of mirroring the normal side onto the faulty side, and in these scenarios, it becomes almost impossible to determine the normal side. ${ }^{5,56}$ However, scientists have proposed formulating a map of the skull that can be used as a template for bilateral reconstruction. Theoretically, it is also possible to construct an ideal craniofacial model from a CT database of a population and can be used as a standard reference for reconstruction. ${ }^{49} \mathrm{~A}$ study by Badiali et al demonstrated the method to construct an average virtual 3-d skull model that could be used as a template to preplan maxillofacial surgeries. Moreover, the template can also be incorporated with a wearable augmented reality that will help surgeons to virtually orient 3-d model to the patient anatomy in real time. ${ }^{57}$ 
Soft tissue deformation and discrepancy in volume between pre-operative and intra-operative images is another problem that has limited the use of navigation in soft tissue correction surgery. Structural image drift is a phenomenon that is caused by the change in the topographic soft tissue landmarks intra-operatively, in contrast to the registered preoperative images. This is because of soft tissue manipulation during surgery that results in an alteration of volume data that was fixed intra-operatively. ${ }^{17,48}$

Other possible factors that are not technologically related but could limit navigation are - the high cost of the equipment and the steep learning curve. The cost of navigation equipment set-up can be expensive for clinics in developing nations. However, considering the system's benefits, it is worth its price to equip our surgical theatre with such a technology gift. ${ }^{5,49}$

\section{Recent Advancement in the Navigation Technology}

In recent years, continuous improvement in modern healthcare has opened up new possibilities for improvising navigation-assisted surgeries to meet the ongoing patientcentered outcomes. To date, there is the top five most recent advancement, reported in the literature that has been made in a navigation technology, which is described briefly in the following subsections.

\section{Intraoperative Augmented Reality (AR)-Based System}

In a traditional navigation set-up, the display is located outside the surgical field. Consequently, surgeons have to precisely coordinate his/her hands' movement on a patient without actually looking at the screen. ${ }^{58}$ On the other hand, while viewing the screen intra-operatively, surgeons have to place instrument arbitrarily on the patients because his/her eyes are fixed on the screen, which is not in the line of the surgical field. Augmented reality system tracker has been introduced to overcome this, which allows surgeons to visualize surgical procedure without moving his/head away from the surgical field. In AR, with the help of a tracker attached to the surgeon's wearable headset, virtual images are converted to real images. This gives surgeons a potential advantage to operate without moving his/ her head away from the surgical site. AR has been further modified with automatic marker-free registration and stereo tracking to minimize the invasiveness of registration marker as with a tradition navigation system..$^{59,60}$

\section{Electromagnetic Microsensors (EM)}

An EM microsensor is a digital chip attached to navigation instruments, and an EM field generator rest under a patient head. The electromagnetic waves generated by the EM field generator are picked up by a digital chip and guide surgeons to register anatomical landmarks more precisely and error-free with the instrument's advantage to be flexible throughout its length. ${ }^{60}$ This unique property of EM has been potentially applied in sinus surgery as it provides surgeons with a roadmap to visualize paranasal sinuses outflow, and ostial dilation can be carried out with minimum morbidity to the patients. Although EM sensors can accurately register landmarks with a minimum error margin of $0.26-0.67 \mathrm{~mm}$, they can be affected by metal distortion. ${ }^{61}$

\section{Ultrasound Guidance Navigation Surgery (US Imaging)}

In US imaging, instead of the registration point marked from the CT scan, the points are marked intra-operatively under the guidance of ultrasound. Usually, in a tradition navigation system, registration is marked based on CT images. However, most of the time, because of the patient's delayed presentation to a surgical team, underlying tissue can be fibrosed and lose its orientation due to the body natural healing mechanism. This can lead to error in point registration. US imaging allows surgeons to register point based on the patient current tissue status intra-operatively, which could minimize the chances of registration failure. ${ }^{62}$

\section{Fast Anatomic Mapping (FAM)}

FAM is a modification of virtual reality (VR) that allows surgeons to visualize $3 \mathrm{~d}$ reconstruction of surgical space continuously. Extensively applied in a cardiac catheter for minimally invasive heart procedures, this technology has been recently incorporated with navigation in tumour ablative surgeries, namely in skull-based surgeries. ${ }^{60}$ FAM helps the surgical team with a virtual representation of the surgical procedures made during oncological surgeries by outlining the tumour mass's border and critical anatomical landmarks in the vicinity.

\section{Haptics Assisted Craniomaxillofacial Surgery}

Is an alternative to the navigation system, which combines stereo visualization with six-degrees-of-freedom. It 
is mainly used in complex maxillofacial trauma cases. It has a high visual-spatial perception and high haptic intuitive feedback in the alignment of bony fragment during fracture reduction. This technology allows surgeons to see patient-specific anatomy in a planning phase and an option of touch, move, rotate objects such as bone fragments and grafts like moving real objects. A wearable device attached to the surgeon's head enable he/she to visualize the entire working volume from different angles by rotating the head in the desired line of the field. ${ }^{63,64}$ Haptics system allows surgeons to perceive critically difficult landmarks such as snap-in fit between the bony segments after fracture reduction. For example, in mandibular fracture cases, assessing the lingual side's reduction, which is impossible to see through naked eyes, can be easily visualized for fixation. Apart from complex trauma cases, haptic technology has also been successfully applied in the field of oncosurgery, particularly in the reconstruction of the fibula osteocutaneous flap. ${ }^{64}$

\section{Conclusion}

Although neurosurgery was the first discipline to introduce navigation technology in their surgical practice, at present maxillofacial surgeons, have well perceived its potential to improve final surgical outcomes. In a nutshell, navigationassisted surgeries can be categorised into - correction of deformity secondary to trauma or craniofacial anomalies, resection of tumour and reconstruction, localisation of foreign bodies, placement of an implant, and correction of the secondary deformity. With better understanding, now this system has gained domain over a majority of the procedures in the head and neck region. Further research and advancement in technology will rectify its limitations and present a tool that will not just be a supplement but sine qua non for surgical practice.

\section{Abbreviations}

CT, computerized tomography; GPS, global positioning device; MRI, magnetic resonance imaging; DICOM, Digital Imaging and Communications in Medicine; AR, Augmented reality; ER, Electromagnetic microsensors; US, Ultrasound guidance; FAM, Fast Anatomic Mapping.

\section{Acknowledgments}

We would like to acknowledge the surgical team from New Hope hospital for introducing us with the Navigation software.

\section{Disclosure}

The authors report no conflicts of interest in this work.

\section{References}

1. Mezger U, Jendrewski C, Bartels M. Navigation in surgery. Langenbeck Arch Surg. 2013;398(4):501-514. doi:10.1007/s00423013-1059-4

2. Dubois L, Schreurs R, Jansen J, et al. Predictability in orbital reconstruction: a human cadaver study. Part II: navigation-assisted orbital reconstruction. J Cranio Maxillofacial Surg. 2015;43(10):2042-2049. doi:10.1016/j.jcms.2015.07.020

3. Dubois L, Essig H, Schreurs R, et al. Predictability in orbital reconstruction. A human cadaver study, part III: implant-oriented navigation for optimized reconstruction. J Cranio Maxillofacial Surg. 2015;43(10):2050-2056. doi:10.1016/j.jcms.2015.08.014

4. Wu Y, Wang F, Huang W, Fan S. Real-time navigation in zygomatic implant placement: workflow. Oral Maxillofacial Surg Clin. 2019;31 (3):357-367. doi:10.1016/j.coms.2019.03.001

5. Sukegawa S, Kanno T, Furuki Y. Application of computer-assisted navigation systems in oral and maxillofacial surgery. Japan Dental Sci Rev. 2018;54(3):139-149. doi:10.1016/j.jdsr.2018.03.005

6. Luebbers HT, Messmer P, Obwegeser JA, et al. Comparison of different registration methods for surgical navigation in cranio-maxillofacial surgery. J Cranio Maxillofacial Surg. 2008;36 (2):109-116. doi:10.1016/j.jcms.2007.09.002

7. Computer assisted surgery. Available from: https://surgeryreference. aofoundation.org/cmf/further-reading/cas-computer-assisted-surgery. Accessed March 18, 2021.

8. Demian N, Pearl C, Woernley TC, Wilson J, Seaman J. Surgical navigation for oral and maxillofacial surgery. Oral Maxillofacial Surg Clin. 2019;31(4):531-538. doi:10.1016/j.coms.2019.06.001

9. Bobek SL. Applications of navigation for orthognathic surgery. Oral Maxillofacial Surg Clin. 2014;26(4):587-598. doi:10.1016/j. coms.2014.08.003

10. Degala S, Shetty SK, Biddappa L. Reconstruction of post-traumatic internal orbital wall defects with titanium mesh. J Maxillofac Oral Surg. 2013;12(4):418-423. doi:10.1007/s12663-012-0444-9

11. Schmelzeisen R, Gellrich NC, Schramm A, Schön R, Otten JE. Navigation-guided resection of temporomandibular joint ankylosis promotes safety in skull base surgery. J Oral Maxillofacial Surg. 2002;60(11):1275-1283. doi:10.1053/joms.2002.35724

12. Hwang K, Kim DH. Analysis of zygomatic fractures. J Craniofacial Surg. 2011;22(4):1416-1421. doi:10.1097/SCS.0b013e31821cc28d

13. Zandi M, Miresmaeili A, Heidari A, Lamei A. The necessity of pterygomaxillary disjunction in surgically assisted rapid maxillary expansion: a short-term, double-blind, historical controlled clinical trial. J Cranio Maxillofacial Surg. 2016;44(9):1181-1186. doi:10.1016/j.jcms.2016.04.026

14. Gong X, He Y, An J, et al. Application of a computer-assisted navigation system (CANS) in the delayed treatment of zygomatic fractures: a randomized controlled trial. J Oral Maxillofacial Surg. 2017;75(7):1450-1463. doi:10.1016/j.joms.2016.10.001

15. Wei JJ, Tang ZL, Liu L, Liao XJ, Yu YB, Jing W. The management of naso-orbital-ethmoid (NOE) fractures. Chin $j$ Traumatol. 2015;18 (5):296-301. doi:10.1016/j.cjtee.2015.07.006

16. Kunz C, Audigé L, Cornelius CP, Buitrago-Téllez CH, Rudderman R, Prein J. The comprehensive AOCMF classification system: orbital fractures-level 3 tutorial. Craniomaxillofac Trauma Reconstr. 2014;7 (1_suppl):92-102. doi:10.1055/s-0034-1389562

17. Yu H, Shen SG, Wang X, Zhang L, Zhang S. The indication and application of computer-assisted navigation in oral and maxillofacial surgery-Shanghai's experience based on 104 cases. J Cranio Maxillofacial Surg. 2013;41(8):770-774. doi:10.1016/j. jcms.2013.01.016 
18. Yang JR, Liao HT. Functional and aesthetic outcome of extensive orbital floor and medial wall fracture via navigation and endoscope-assisted reconstruction. Ann Plast Surg. 2019;82(1S): S77-S85. doi:10.1097/SAP.0000000000001700

19. He D, Li Z, Shi W, et al. Orbitozygomatic fractures with enophthalmos: analysis of 64 cases treated late. J Oral Maxillofacial Surg. 2012;70(3):562-576. doi:10.1016/j.joms.2011.02.041

20. Yu H, Shen G, Wang X, Zhang S. Navigation-guided reduction and orbital floor reconstruction in the treatment of zygomatic-orbitalmaxillary complex fractures. J Oral Maxillofacial Surg. 2010;68 (1):28-34. doi:10.1016/j.joms.2009.07.058

21. Hallikainen D, Iizuka T, Lindqvist C. Cross-sectional tomography in evaluation of patients undergoing sagittal split osteotomy. J Oral Maxillofacial Surg. 1992;50(12):1269-1273. doi:10.1016/02782391(92)90225-O

22. Kang SH, Kim MK, Choi YS, Park W, Lee SH. Navigation-assisted intraoral vertical ramus osteotomy. J Oral Maxillofacial Surg. 2011;69(3):931-934. doi:10.1016/j.joms.2010.05.021

23. d'Hauthuille C, Taha F, Devauchelle B, Testelin S. Comparison of two computer-assisted surgery techniques to guide a mandibular distraction osteogenesis procedure Technical note. Int J Oral Maxillofac Surg. 2005;34(2):197-201. doi:10.1016/j.ijom.2004.04.001

24. Badiali G, Roncari A, Bianchi A, Taddei F, Marchetti C, Schileo E. Navigation in orthognathic surgery: 3D accuracy. Facial Plastic Surg. 2015;31(05):463-473. doi:10.1055/s-0035-1564716

25. Shiba H, Kato S, Nakagawa T. Use of navigation systems in orthognathic surgery. Int J Oral Maxillofac Surg. 2015;44:e288. doi:10.1016/j.ijom.2015.08.319

26. Voss PJ, Leow AM, Schulze D, Metzger MC, Liebehenschel N, Schmelzeisen R. Navigation-guided resection with immediate functional reconstruction for high-grade malignant parotid tumour at skull base. Int J Oral Maxillofac Surg. 2009;38(8):886-890. doi:10.1016/j. ijom.2009.04.007

27. Komune N, Matsushima K, Matsuo S, Safavi-Abbasi S, Matsumoto N, Rhoton AL. The accuracy of an electromagnetic navigation system in lateral skull base approaches. Laryngoscope. 2017;127(2):450-459. doi:10.1002/lary.25998

28. Copeland BJ, Senior BA, Buchman CA, Pillsbury III HC. The accuracy of computer-aided surgery in neurotologic approaches to the temporal bone: a cadaver study. Otolaryngology Head Neck Surg. 2005;132(3):421-428. doi:10.1016/j.otohns.2004.10.013

29. Gunkel AR, Vogele M, Martin A, Bale RJ, Thumfart WF, Freysinger W. Computer-aided surgery in the petrous bone. Laryngoscope. 1999;109(11):1793-1799. doi:10.1097/00005537199911000-00013

30. Schramm A, Gellrich NC, Gutwald R, et al. Indications for computerassisted treatment of cranio-maxillofacial tumors. Computer Aided Surg. 2000;5(5):343-352. doi:10.3109/10929080009149852

31. Schramm A, Suarez-Cunqueiro MM, Barth EL, et al. Computerassisted navigation in craniomaxillofacial tumors. J Craniofacial Surg. 2008;19(4):1067-1074. doi:10.1097/SCS.0b013e3181760fc0

32. Feichtinger M, Pau M, Zemann W, Aigner RM, Kärcher H. Intraoperative control of resection margins in advanced head and neck cancer using a 3D-navigation system based on PET/CT image fusion. J Cranio Maxillofacial Surg. 2010;38(8):589-594. doi:10.1016/j.jcms.2010.02.004

33. Gangloff P, Mastronicola R, Cortese S, et al. Navigation in head and neck oncological surgery: an emerging concept. Revue de laryngologie-otologie-rhinologie. 2011;132(4-5):203-207.

34. Rana M, Essig H, Eckardt AM, et al. Advances and innovations in computer-assisted head and neck oncologic surgery. J Craniofacial Surg. 2012;23(1):272-278. doi:10.1097/SCS.0b013e318241bac7

35. Zhu JH, Yang R, Guo YX, Wang J, Liu XJ, Guo CB. Navigationguided core needle biopsy for skull base and parapharyngeal lesions: a five-year experience. Int J Oral Maxillofac Surg. 2020;50(1):7-13. doi:10.1016/j.ijom.2020.05.007
36. Bell RB. Computer planning and intraoperative navigation in cranio-maxillofacial surgery. Oral Maxillofacial Surg Clin. 2010;22 (1):135-156. doi:10.1016/j.coms.2009.10.010

37. Bell RB, Weimer KA, Dierks EJ, Buehler M, Lubek JE. Computer planning and intraoperative navigation for palatomaxillary and mandibular reconstruction with fibular free flaps. J Oral Maxillofacial Surg. 2011;69(3):724-732. doi:10.1016/j.joms.2009.12.040

38. Nkenke E, Neukam FW. Autogenous bone harvesting and grafting in advanced jaw resorption: morbidity, resorption and implant survival. Eur J Oral Implantol. 2014;7(Suppl 2):S203-S217.

39. Campbell A, Costello BJ. Retrieval of a displaced third molar using navigation and active image guidance. J Oral Maxillofacial Surg. 2010;68(2):480-485. doi:10.1016/j.joms.2009.06.032

40. Wei R, Xiang-Zhen L, Bing G, Da-long S, Ze-Ming T. Removal of a foreign body from the skull base using a customized computer-designed guide bar. J Cranio Maxillofacial Surg. 2010;38 (4):279-283. doi:10.1016/j.jcms.2009.07.006

41. Sießegger M, Mischkowski RA, Schneider BT, Krug B, Klesper B, Zöller JE. Image guided surgical navigation for removal of foreign bodies in the head and neck. J Cranio Maxillofacial Surg. 2001;29 (6):321-325. doi:10.1054/jcms.2001.0254

42. Lee TY, Zaid WS. Broken dental needle retrieval using a surgical navigation system: a case report and literature review. Oral Surg Oral Med Oral Pathol Oral Radiol. 2015;119(2):e55-e59. doi:10.1016/j. oooo.2014.08.019

43. Eggers G, Haag C, Hassfeld S. Image-guided removal of foreign bodies. $\mathrm{Br} J$ Oral Maxillofacial Surg. 2005;43(5):404-409. doi:10.1016/j.bjoms.2005.01.016

44. Gerbino G, Zavattero E, Berrone M, Berrone S. Management of needle breakage using intraoperative navigation following inferior alveolar nerve block. J Oral Maxillofacial Surg. 2013;71 (11):1819-1824. doi:10.1016/j.joms.2013.07.023

45. Stein KM. Use of intraoperative navigation for minimally invasive retrieval of a broken dental needle. J Oral Maxillofacial Surg. 2015;73(10):1911-1916. doi:10.1016/j.joms.2015.04.033

46. Özer N, Üçem F, Saruhanoğlu A, Yilmaz S, Tanyeri H. Removal of a maxillary third molar displaced into pterygopalatine fossa via intraoral approach. Case Rep Dent. 2013;2013:2013. doi:10.1155/ 2013/392148

47. Blondeau F, Daniel NG. Extraction of impacted mandibular third molars: postoperative complications and their risk factors. $J$ Can Dent Assoc (Tor). 2007;73:4.

48. Panchal N, Mahmood L, Retana A, Emery R. Dynamic navigation for dental implant surgery. Oral Maxillofacial Surg Clin. 2019;31 (4):539-547. doi:10.1016/j.coms.2019.08.001

49. Emery RW, Korj O, Agarwal R. A review of in-office dynamic image navigation for extraction of complex mandibular third molars. J Oral Maxillofacial Surg. 2017;75(8):1591-1600. doi:10.1016/j. joms.2017.03.031

50. Kasten B, Arastu A, Panchal N. Dental implant surgery: from conventional to guided to navigated approach. Curr Oral Health Rep. 2018;5(2):140-146. doi:10.1007/s40496-018-0182-2

51. Miller RJ, Bier J. Surgical navigation in oral implantology. Implant Dent. 2006;15(1):41-47. doi:10.1097/01.id.0000202637.61180.2b

52. Aydemir CA, Arisan V. Accuracy of dental implant placement via dynamic navigation or the freehand method: a split-mouth randomized controlled clinical trial. Clin Oral Implants Res. 2020;31 (3):255-263. doi:10.1111/clr.13563

53. Elian N, Jalbout ZN, Classi AJ, Wexler A, Sarment D, Tarnow DP. Precision of flapless implant placement using real-time surgical navigation: a case series. Int $J$ Oral Maxillofacial Implants. 2008;23:6.

54. Siessegger M, Schneider BT, Mischkowski RA, et al. Use of an image-guided navigation system in dental implant surgery in anatomically complex operation sites. J Cranio Maxillofacial Surg. 2001;29(5):276-281. doi:10.1054/jcms.2001.0242 
55. Wu D, Zhou L, Yang J, et al. Accuracy of dynamic navigation compared to static surgical guide for dental implant placement. Int J Implant Dentistry. 2020;6(1):1-8. doi:10.1186/s40729-02000272-0

56. Azarmehr I, Stokbro K, Bell RB, Thygesen T. Surgical navigation: a systematic review of indications, treatments, and outcomes in oral and maxillofacial surgery. J Oral Maxillofacial Surg. 2017;75 (9):1987-2005. doi:10.1016/j.joms.2017.01.004

57. Badiali G, Marcelli E, Bortolani B, Marchetti C, Cercenelli L. An average three-dimensional virtual human skull for a template-assisted maxillofacial surgery. Int $J$ Artif Organs. 2019;42(10):566-574. doi:10.1177/0391398819849075

58. Bosc R, Fitoussi A, Hersant B, Dao TH, Meningaud JP. Intraoperative augmented reality with heads-up displays in maxillofacial surgery: a systematic review of the literature and a classification of relevant technologies. Int J Oral Maxillofac Surg. 2019;48(1):132-139. doi:10.1016/j.ijom.2018.09.010

59. Ayoub A, Pulijala Y. The application of virtual reality and augmented reality in Oral \& Maxillofacial Surgery. BMC Oral Health. 2019;19 (1):1-8. doi:10.1186/s12903-019-0937-8
60. Schmale IL, Vandelaar LJ, Luong AU, Citardi MJ, Yao WC. Imageguided surgery and intraoperative imaging in rhinology: clinical update and current state of the art. Ear Nose Throat J. 2020;0145561320928202.

61. Nafis C, Jensen V, Beauregard L, Anderson P. Method for estimating dynamic EM tracking accuracy of surgical navigation tools. In Medical Imaging 2006: visualization, Image-Guided Procedures, and Display 2006 Mar 10 (Vol. 6141, p. 61410K). Int Soc Optics Photonics.

62. Chen X, Bao N, Li J, Kang Y A review of surgery navigation system based on ultrasound guidance. In: 2012 IEEE International Conference on Information and Automation; Jun 6, 2012; IEEE. 882-886.

63. Olsson P, Nysjö F, Hirsch JM, Carlbom IB. A haptics-assisted cranio-maxillofacial surgery planning system for restoring skeletal anatomy in complex trauma cases. Int J Comput Assist Radiol Surg. 2013;8(6):887-894. doi:10.1007/s11548-013-0827-5

64. Olsson P. Haptics with applications to cranio-maxillofacial surgery planning. Doctoral dissertation, Acta Universitatis Upsaliensis; 2015.
Clinical, Cosmetic and Investigational Dentistry

\section{Publish your work in this journal}

Clinical, Cosmetic and Investigational Dentistry is an international, peer-reviewed, open access, online journal focusing on the latest clinical and experimental research in dentistry with specific emphasis on cosmetic interventions. Innovative developments in dental materials, techniques and devices that improve outcomes and patient

\section{Dovepress}

satisfaction and preference will be highlighted. The manuscript management system is completely online and includes a very quick and fair peer-review system, which is all easy to use. Visit

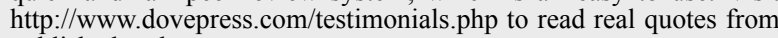
published authors. 